\title{
Damping Analyses of Structural Vibrations and Shunted Piezoelectric Transducers
}

\author{
Saber Mohammadi and Akram Khodayari \\ Mechanical Engineering Department, Razi University, 6714967346 Kermanshah, Iran \\ Correspondence should be addressed to Saber Mohammadi, saberm7@yahoo.com
}

Received 17 November 2011; Accepted 7 December 2011

Academic Editor: Tao Li

Copyright ( $) 2012$ S. Mohammadi and A. Khodayari. This is an open access article distributed under the Creative Commons Attribution License, which permits unrestricted use, distribution, and reproduction in any medium, provided the original work is properly cited.

\begin{abstract}
Piezoelectric transducers in conjunction with appropriate electric networks can be used as a mechanical energy dissipation device. Alternatively, undesired mechanical energy of a structure could be converted into electrical energy that can be dissipated through a shunt network in the form of Joule heating. This paper presents an experimental method to calculate damping energy in mechanical systems. However, the mathematical description of damping mechanism is much more complicated, and any process responsible for the occurrence of damping is very intricate. Structural and piezoelectric damping are calculated and analysed in the case of pulse switching or SSDI semiactive vibration control technique. This technique which was developed in the field of piezoelectric damping consists in triggering the inverting switch on each extremum of the piezoelectric voltage which induces an increase of the electromechanical energy conversion.
\end{abstract}

\section{Introduction}

Vibration damping is one of the manifestations of mechanical energy dissipation related to motion in mechanical systems. Damping processes have been studied for a long time. Damping forces are small compared to the other interactions in a mechanical system and yet their mathematical description remains much more complicated. Actually, any process responsible for the occurrence of damping is very intricate and the knowledge of it is insufficient. Sometimes just changing the system's stiffness or mass to alter the resonance frequencies can reduce the unwanted vibration as long as the excitation frequencies do not change. But in most cases, the vibrations need to be dissipated using damping materials or devices that are tuneable with vibration.

Several methods have been investigated in case of vibration damping. These methods have the forms of passive, semiactive, and active treatments which can be used for sound/vibration cancellation. Active control involves the use of active elements (actuators) along with sensors and controllers (analogue or digital) to produce an out-ofphase actuation to cancel the disturbance causing the noise/vibration [1]. All other methods that do not include a real-time active algorithm can be grouped under the passive control option. Passive damping refers to energy dissipation within the structure by add-on damping devices. Viscous dampers (dashpots), viscoelastic damping, tunedmass dampers, dynamic absorbers, and shunted piezoelectric dampers are the mechanisms of passive vibration control. The most common types of passive damping treatments of viscoelastic materials were described by Rao [2]. Tuned dampers are the reactive devices which used to oscillation damping at a particular resonant frequency. It consists of an inertia element, a compliant/resilient element, and an energy dissipating element. The inertial, resilient, and dissipative elements in such devices are: mass, spring and dashpot. Depending on the application, these devices are sized from a few grams to many tons. They attract the vibration energy of the target mode and dissipate it as heat through the action of its dashpot.

Electronic damping using piezoelectric ceramics (PiezoShunt) is less temperature sensitive and more tuneable compared with viscoelastic damping treatments. In this damping technique, the mechanical energy of the structure is converted to electrical energy by piezoelectric material. The electrical energy, in turn is dissipated, as heat, in an electrical 
shunt circuit. These methods are interesting because they do not rely on any operative energy as in active control. They consist in the drive of a few solid-state switches (i.e., MOSFET transistor) requiring very few power and, in general, are simple to implement. Pulse switching damping technique [3-7] which is implemented in this paper consists in leaving the piezo elements in open-circuit except during very brief period of time where the electric charge is either suppressed in a short circuit or inverted on an inductance.

Many various terms are used to represent vibration damping. These representations merely indicate the mathematical model used to represent the physical mechanism of damping that is still not clearly understood for many cases $[8,9]$. The purpose of this paper is the practical measurement of damping energy which is the vital problem in vibrating systems. The effects of piezoelectric damping and structural damping are observed experimentally and compared with each other. At the following, the damping behaviors in low and high values of deformation are studied. In order to analyse the vibration damping, a multimodal structure equipped with piezoelectric elements wired on a pulse switching cell is experimented.

\section{Energy Analyses and Energetic Considerations}

In vibration analyses, it is concerned with damping in terms of system response. The loss of energy from the oscillatory system results in the decay of free vibration amplitude. In steady-state forced vibration, the loss of energy is balanced by the energy which is supplied by the excitation. Energy dissipation is usually determined under conditions of cyclic oscillations. The energy dissipated per cycle due to a damping force $F_{d}$ is computed from the general equation [8]

$$
W_{d}=\oint F_{d} d u
$$

where $u$ is the displacement. On the other hand, experiments by several investigators [8] indicate that for most structural metals (such as steel), the energy dissipated per cycle (structural damping) proportional to the squared of the vibration amplitude and independent of the frequency over a wide frequency range. Then energy dissipated by structural damping may be written as [10]

$$
W_{d}=\alpha_{d} u_{\max }^{2}
$$

where $\alpha_{d}$ is a constant independent of the frequency of harmonic oscillation and $u_{\max }$ is the amplitude of vibration. Material in cyclic stress exhibits a stress-strain relation characterized by a hysteresis loop. The cyclic dissipated energy is proportional to the hysteresis loop area. Consequently, the energy balance equation based on hysteresis loop can be deduced. The following is an equation of motion of a vibrating system

$$
\ddot{u}=F_{i}(u, \dot{u})+F_{e}(t),
$$

where $F_{i}(u, \dot{u})$ and $F_{e}(t)$ correspond to internal and external forces per unit mass, respectively. The external and the internal loops (hysteresis loops) can be then described by

$$
F_{e}=F_{e}(u), \quad F_{i}=F_{i}(u) .
$$

We can write down the following relationship between them:

$$
F_{i}(u)=\ddot{u}(u)-F_{e}(u) .
$$

Owing to this relationship we, can convert the external loop into an internal one by subtracting the values corresponding to the external loop from acceleration dependent on displacement. Multiplying (5) by $d u=\dot{u} d t$ enables one to obtain the infinitesimal work done by exciting forces. Integrating over the entire period leads to

$$
\int_{0}^{T} F_{i}[u(t)] \dot{u}(t) d t=\int_{0}^{T} \ddot{u}(t) \dot{u}(t) d t-\int_{0}^{T} F_{e}[u(t)] \dot{u}(t) d t .
$$

The first integral on the right-hand side of (6) equals zero for periodic motion. Hence, we can derive the following wellknown formula:

$$
L i=L e,
$$

which say that dissipated energy is equal to the work done by external forces $[11,12]$.

The experimental sample in this paper is a cantilever beam equipped with piezoelectric patches wired on a pulse switching cell (Figure 1) [13].

The total outgoing current from the piezoelectric patches using their constitutive equations can be calculated as [14, 15]

$$
I=\sum_{i=1}^{N} \alpha_{i} \dot{q}_{i}-C_{p} \dot{v}
$$

where $C_{p}$ is the capacitance of the piezoelectric elements. In general, the piezoelectric patches are wired together in parallel. Multiplying each term of (8) by the voltage and integrating over the time shows that the converted energy is the sum of the electrostatic energy stored on the piezoelectric elements and the energy absorbed or dissipated by the electrical device as

$$
E_{t}=\sum_{i=1}^{N} \alpha_{i} \int_{0}^{t} \dot{q}_{i} v_{i} d t=\frac{1}{2} C_{p} v^{2}+\int_{0}^{t} v I d t
$$

In the case of pulse switching technique, an electric circuit is connected to the piezoelectric elements. This circuit can be used for dissipating energy from the system or energy recovery or for both of them. It consists of a switching device in parallel with the piezoelectric elements. The current in the switching device is always zero except during the voltage inversion that takes place at each switch trigger. At each inversion, the energy extracted from the piezoelement is equal to the difference in the electrostatic energy on the piezoelectric elements before and after the voltage inversion 


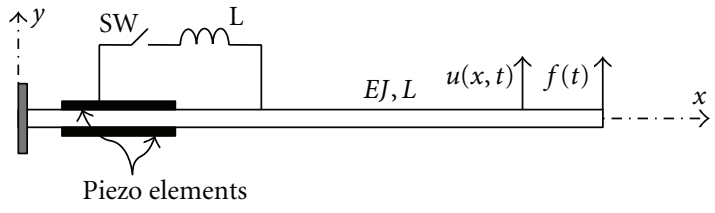

Figure 1: Cantilever beam where $u(x, t)$ is the beam deflection along the transverse direction $(y)$ and $f(t)$ is the excitation force.

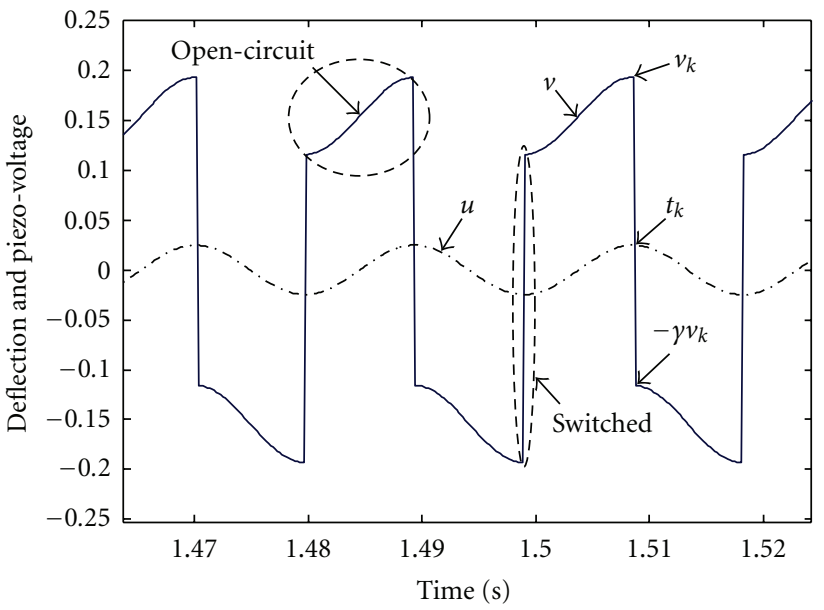

Figure 2: Strategy of pulse switching technique.

jump (Figure 2). The energy dissipated in the switching device is then given by

$$
\int_{0}^{t} v I d t=\frac{1}{2} C_{p} \sum_{k} v_{k}^{2}\left(1-\gamma^{2}\right),
$$

where $v_{k}$ is the piezoelectric voltage just before the $k$ th inversion and $\gamma$ is the inversion coefficient.

There are two types of damping: material damping (structural) and system damping. Material damping is the damping inherent in the material, while system damping includes the damping at adds damper devices to the system (such as, piezoelectric material), in addition to material damping. By calculating damping energy (7) in the case of forced harmonic vibration during a time period for controlled and uncontrolled cases, respectively, the piezoelectric damping can be calculated. Among to the methods of damping measurement of a vibrating system, attenuation [11] is the other parameter to describe the vibration damping as

$$
\text { Attenuation }=20 \log \left(\frac{\left(u_{\max }\right)_{c}}{\left(u_{\max }\right)_{\mathrm{unc}}}\right),
$$

where $\left(u_{\max }\right)_{c}$ and $\left(u_{\max }\right)_{\text {unc }}$ are the maximum amplitude of displacement in controlled (structure with piezo elements) and uncontrolled cases, respectively.

\section{Experimental Results}

Experimental setup considered is a steel beam equipped with piezoelectric inserts. This structure corresponds to the
TABLE 1: Characteristics of the experimental structure.

\begin{tabular}{lc}
\hline Plate material & Steel \\
Plate dimensions (free) & $180 \times 90 \times 2 \mathrm{~mm}^{3}$ \\
Piezoelectric material & P189 \\
Number of piezo elements & 12 (on each face) \\
Piezo elements position & $10 \mathrm{~mm}$ from the clamping end \\
Piezo elements dimensions & $10 \times 30 \times 0.3 \mathrm{~mm}^{3}$ \\
Open-circuit resonance frequency & $56.48 \mathrm{~Hz}$ \\
Inversion coefficient $\gamma$ & 0.6 \\
\hline
\end{tabular}

description given in Table 1. The proposed control strategy is implemented using a laboratory PC-based real-time DSP controller environment. Either the voltage or the deflection signals are sampled by the board as input data. According to the proposed method, the switch trigger is generated by the digital output of the control board, connected on a pulse switching device as described in [5]. The displacement sensor used is a simple piezoelectric insert collocated with the main inserts. The sensor thickness and material are similar to the control insert and generates, therefore, the same open-circuit voltage. Control strategy programming and implementation is done using the Matlab/Simulink software environment and dSPACE Real-Time Workshop for real-time computing and input/output control. Excitation of the beam is obtained using an electromagnet driven using an audio amplifier, by a harmonic excitation generated by the dSPACE board.

In practice, to measure the energy dissipated per cycle, using the concept of (7), the procedure is rather straightforward. For a cantilever beam with applied $f(t)$ at its free-end, this energy loss is given by the integral over a complete cycle in the case of harmonic excitation as

$$
E_{f}=\int_{0}^{T} f(t) \dot{u} d t
$$

where $T$ is the cycle's period. This integration can be estimated by using $Z$ data points separated by $\Delta t$ seconds, so that digital summation over $Z-1$ data points replaces the continuous integral. Thus (12) leads to

$$
E_{f}=\frac{1}{n} \int_{0}^{n T} f(t) \dot{u} d t=\frac{\Delta t}{n} \sum_{i=1}^{Z-1}(f \dot{u})_{i}
$$

where $n$ is the number of cycles. Therefore, by multiplying generated force in the dSPACE board to the free-end measured velocity by the laser sensor and dSPACE step time $\Delta t$ for the $Z$ captured data points during $n$ cycle, the dissipated energy from (13) can be calculated during a time period. Piezoelectric damping energy is equal to the difference between controlled and uncontrolled cases.

Figure 3 shows the variations of capture (displacement sensor) signal amplitude versus the excitation force amplitude in controlled and uncontrolled cases, respectively. The curves slope indicate the influence coefficient (inverse of stiffness) at the point that in which excitation force is applied (It should be mentioned that the capture signal is an image of the free-end deflection of beam; it has 


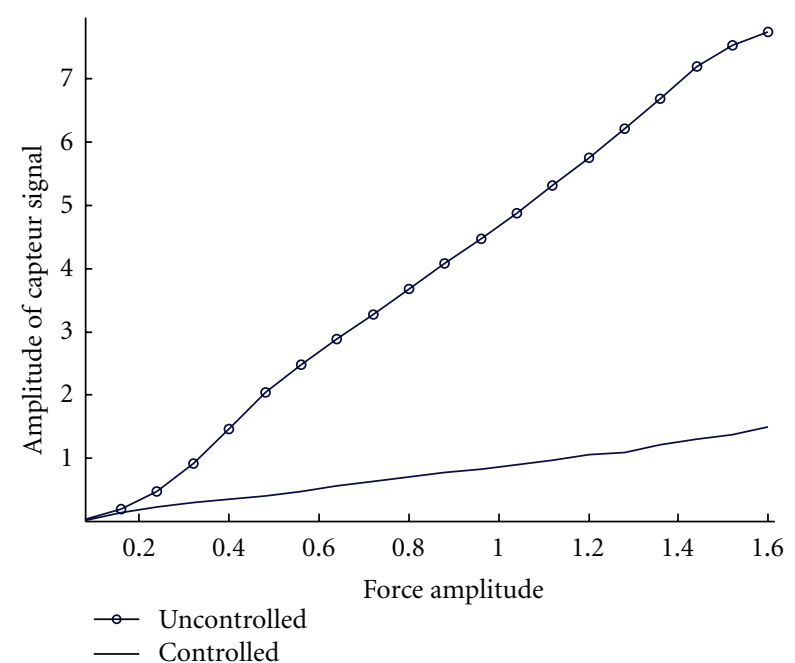

FIGURE 3: Variations of the capture signal amplitude versus the force amplitude in controlled and uncontrolled cases.

placed at the clamped end of beam that in which the bending moment is maximum and consequently the capture voltage (piezoelement deformation) and free-end deflection are maximum. Indeed, computations have been made using the image of deflection given by the collocated piezoelectric deflection sensor.). Damping effect increases gradually with increase of force amplitude which is more evident in controlled case because of the piezoelectric damping. Piezoelectric effect is too little in small deformations. The vertical distance between these two curves show the effect of piezoelectric damping. Since the structural damping is the same in controlled (piezo elements) and uncontrolled cases, the difference of curves slope is just the one caused by the piezoelectric damping. In high deformations (force amplitude $>1.4$ ), the curves tend to be parallel with each other. It is mentioned that the maximum damping caused by piezoelectric elements was limited and have its ultimate value. After the ultimate value, the curves rise similarly with increase of force amplitude. In this case, the additional injected energy to the system only increases the amplitude of vibration in two cases (controlled and uncontrolled) proportionally. In this figure, the excitation frequency is equal to the first resonant frequency of the beam (Table 1).

Figure 4 shows the variations of capture signal attenuation versus the force amplitude. From the figure, the decreasing of the attenuation was limited. The minimum attenuation (ultimate value) is about $-10 \mathrm{~dB}$. It is observed that after the ultimate value the curve rise slowly with increase of force amplitude. In this case, structural damping is more predominant than piezoelement damping (It is proportional to the square of vibration amplitude, (2).). Also, the excitation frequency is the first resonant frequency (Table 1).

Figure 5 indicates the variations of periodical damping energy versus the square of capture signal amplitude. Damping energy in controlled case increases faster than one in uncontrolled case with increase of capture signal amplitude.

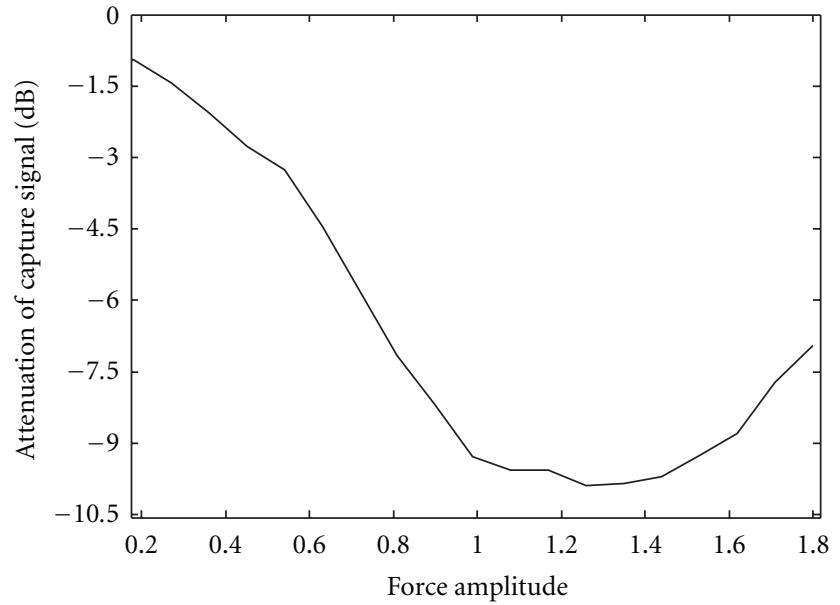

Figure 4: Variations of the capture signal attenuation in $\mathrm{dB}$ versus the force amplitude.

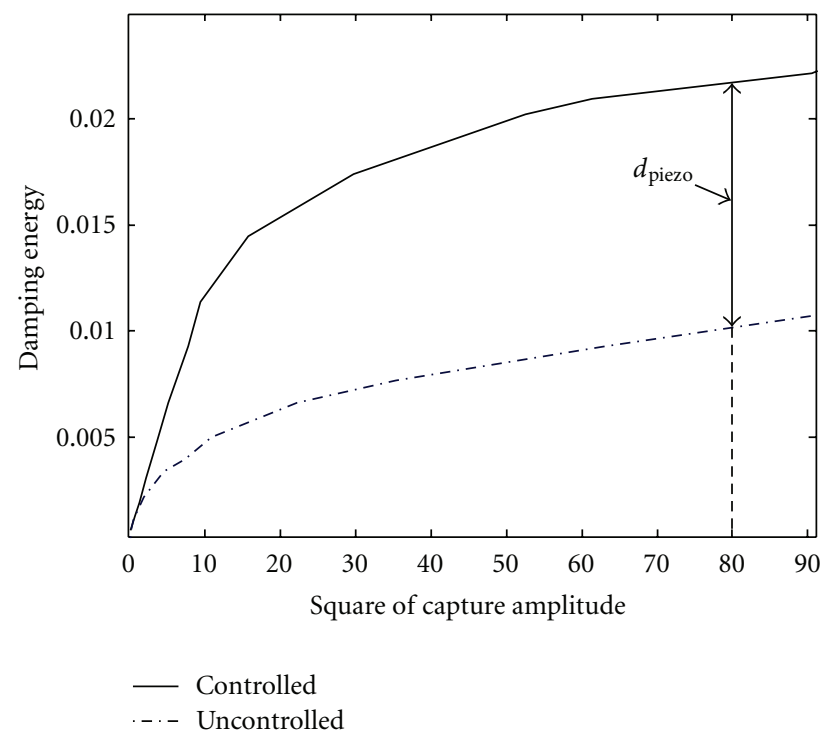

FIGURE 5: Variations of damping energy versus the square of capture signal amplitude.

Therefore, the curve slope in controlled case is more than the one in uncontrolled case up to the value of 60 . After that, the structural damping is more predominant, and piezoelectric damping reaches its ultimate value. Then, two curves are approximately parallel (with distance $d_{\text {piezo }}$ ). It can be concluded that (2) holds true only for high values of structural deformation (The curves slope are constant in high deformations). Also, the values of $\alpha_{d}$ are the same for the two cases (controlled and uncontrolled) and are just as the one for structural damping. Piezoelectric effect increases damping only more than uncontrolled case equal $d_{\text {piezo }}$. Thus as (2) is relevant to structural damping, this equation for controlled case is

$$
W_{d}=\alpha_{d} u_{\max }^{2}+d_{\text {piezo }} .
$$


The slop of these curves gives the value of $\alpha_{d}$ and is approximately equal to 0.026 .

\section{Conclusions}

This paper showed an experimental method to calculate the damping energy in mechanical system. However, the mathematical description of damping mechanism is much more complicated and any process responsible for the occurrence of damping is very intricate and the knowledge of it is insufficient. Structural damping and piezoelectric damping for pulse switching semiactive nonlinear control technique have been calculated and analysed. Pulse switching control technique is interesting for structural damping applications because it presents simultaneously good damping performances, a good robustness, and very low power requirements. Finally, it is important to consider that this technique is simple enough to be self-powered. Piezoelectric damping will increase with increase of force amplitude but its maximum value was limited and has its ultimate value.

\section{Acknowledgments}

The authors would like to thank Professor D. Guyomar and Professor C. Richard from LGEF-INSA for their kind help.

\section{References}

[1] D. Niederberger, A. Fleming, S. O.R. Moheimani, and M. Morari, "Adaptive multi-mode resonant piezoelectric shunt damping," Smart Materials and Structures, vol. 13, no. 5, pp. 1025-1035, 2004.

[2] M. D. Rao, "Recent applications of viscoelastic damping for noise control in automobiles and commercial airplanes," Journal of Sound and Vibration, vol. 262, no. 3, pp. 457-474, 2003.

[3] A. Badel, Récupération d'énergie et contrôle vibratoire par éléments piézoélectriques suivant une approche non linéaire, Ph.D. thesis, Ecole Doctorale de l'Université de Savoie, 2005.

[4] D. Guyomar and A. Badel, "Nonlinear semi-passive multimodal vibration damping: an efficient probabilistic approach," Journal of Sound and Vibration, vol. 294, no. 1-2, pp. 249-268, 2006.

[5] C. Richard, D. Guyomar, D. Audigier, and G. Bassaler, "Enhanced semi passive damping using continuous switching of a piezoelectric device on an inductor," in Smart Structures and Materials, vol. 3989 of Proceedings of SPIE, pp. 288-299, San Diego, Calif, USA, 2000.

[6] C. Richard, D. Guyomar, D. Audigier, and G. Ching, "Semi passive damping using continuous switching of a piezoelectric device," in Smart Structures and Materials, vol. 3672 of Proceedings of SPIE, p. 104, San Diego, Calif, USA, 1998.

[7] L. R. Corr and W. W. Clark, "A novel semi-active multi-modal vibration control law for a piezoceramic actuator," Journal of Vibration and Acoustics, Transactions of the ASME, vol. 125, no. 2, pp. 214-222, 2003.

[8] W. T. Thomson, Theory of Vibration with Applications, Prentice-Hall, Upper Saddle River, NJ, USA, 1981.

[9] L. Meirovitch, Elements of Vibrations Analyses, McGraw-Hill, New York, NY, USA, 1986.

[10] L. Meirovitch, Analytical Methods in Vibrations, The Macmillan Company, New York, NY, USA, 1967.
[11] Z. Osinski, Damping of Vibrations, A.A. Balkema, Rotterdam, The Netherlands, 1998.

[12] Z. Osinski, "Hysteresis loops of the vibrating system," Machine Dynamics Problems, vol. 5, 1993.

[13] D. Guyomar, C. Richard, and S. Mohammadi, "Semi-passive random vibration control based on statistics," Journal of Sound and Vibration, vol. 307, no. 3-5, pp. 818-833, 2007.

[14] A. Khodayari, A. Ahmadi, and S. Mohammadi, "On physical realization of the wireless semi active real time vibration control based on signal statistical behavior," Sensors and Actuators, A, vol. 167, no. 1, pp. 102-109, 2011.

[15] S. Mohammadi, Semi-passive vibration control using shunted piezoelectric materials and harvesting energy, Ph.D. thesis, INSA, Lyon, France, 2007. 

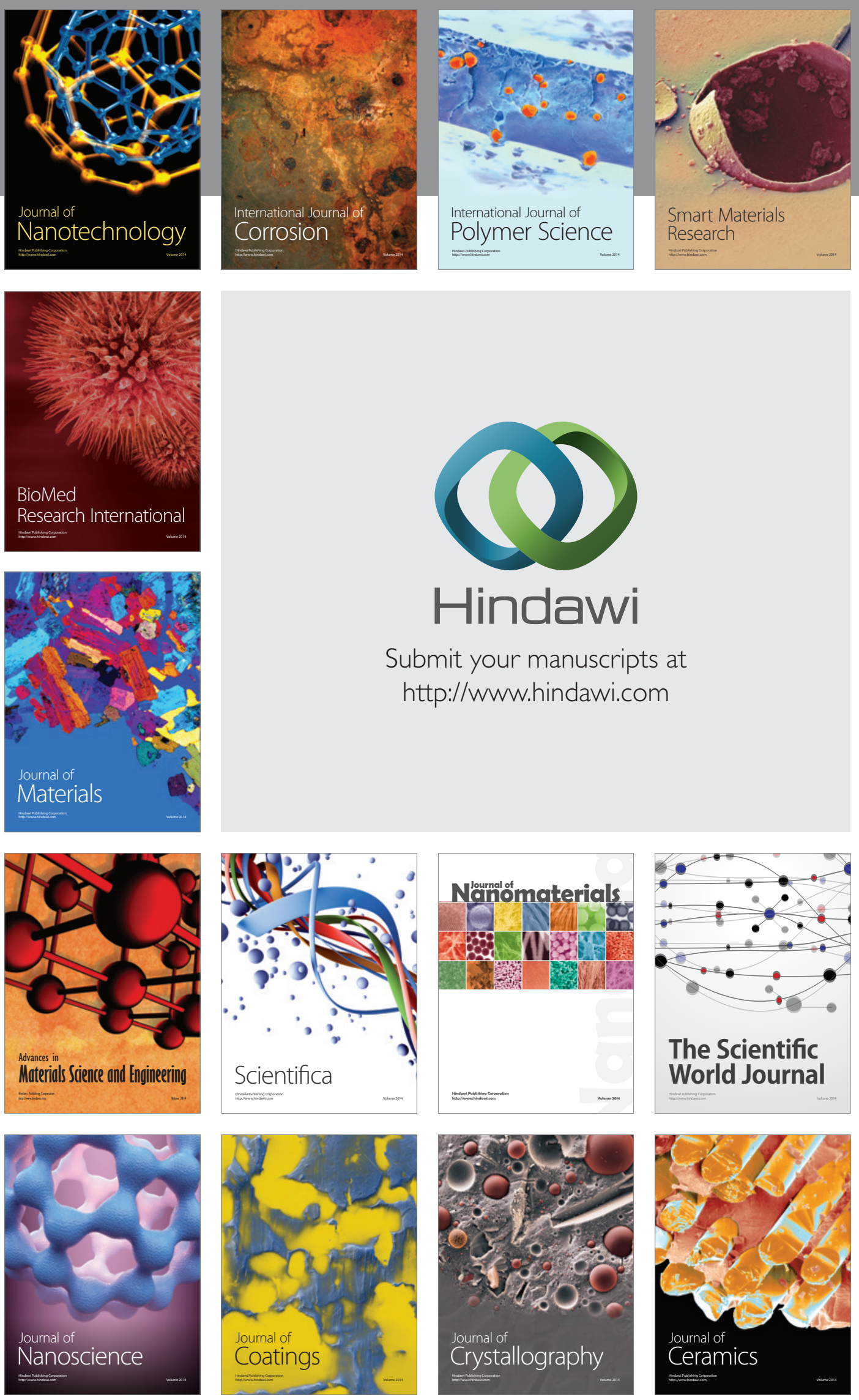

The Scientific World Journal

Submit your manuscripts at

http://www.hindawi.com

\section{World Journal}

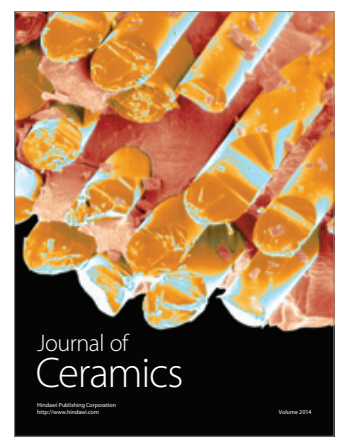

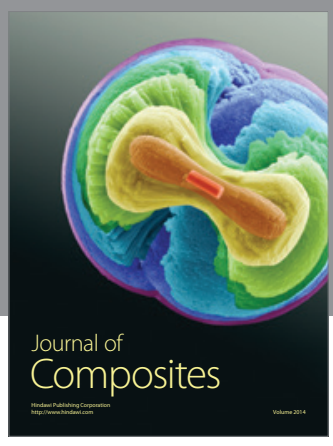
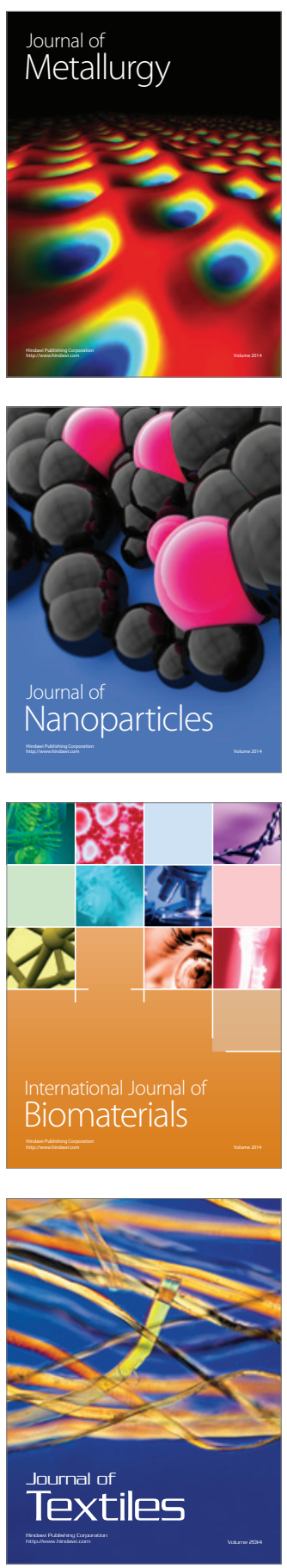